IJMMS 31:6 (2002) 321-327

PII. S0161171202107149

http://ijmms.hindawi.com

(c) Hindawi Publishing Corp.

\title{
SUBMODULES OF SECONDARY MODULES
}

\section{SHAHABADDIN EBRAHIMI ATANI}

Received 31 July 2001 and in revised form 25 January 2002

Let $R$ be a commutative ring with nonzero identity. Our objective is to investigate representable modules and to examine in particular when submodules of such modules are representable. Moreover, we establish a connection between the secondary modules and the pure-injective, the $\Sigma$-pure-injective, and the prime modules.

2000 Mathematics Subject Classification: 13F05.

1. Introduction. In this paper, all rings are commutative rings with identity and all modules are unital. The notion of associated prime ideals and the related one of primary decomposition are classical. In a dual way, we define the attached prime ideals and the secondary representation. This theory is developed in the appendix to Section 6 in Matsumura [6] and in Macdonald [5]. Now we define the concepts that we will need.

Let $R$ be a ring and let $0 \neq M$ be an $R$-module. Then $M$ is called a secondary module (second module) provided that for every element $r$ of $R$ the homothety $M \stackrel{r}{\longrightarrow} M$ is either surjective or nilpotent (either surjective or zero). This implies that nilrad $(M)=$ $P\left(\operatorname{Ann}(M)=P^{\prime}\right)$ is a prime ideal of $R$, and $M$ is said to be $P$-secondary ( $P^{\prime}$-second), so every second module is secondary (the concept of second module is introduced by Yassemi [14]). A secondary representation for an $R$-module $M$ is an expression for $M$ as a finite sum of secondary modules (see [5]). If such a representation exists, we will say that $M$ is representable.

If $R$ is a ring and $N$ is a submodule of an $R$-module $M$, the ideal $\{r \in R: r M \subseteq N\}$ will be denoted by $(N: M)$. Then $(0: M)$ is the annihilator of $M, \operatorname{Ann}(M)$. A proper submodule $N$ of a module $M$ over a ring $R$ is said to be prime submodule (primary submodule) if for each $r \in R$ the homothety $M / N \stackrel{r \cdot}{\longrightarrow} M / N$ is either injective or zero (either injective or nilpotent), so $(0: M / N)=P\left(\operatorname{nilrad}(M / N)=P^{\prime}\right)$ is a prime ideal of $R$, and $N$ is said to be $P$-prime submodule ( $P^{\prime}$-primary submodule). So $N$ is prime in $M$ if and only if whenever $r m \in N$, for some $r \in R, m \in M$, then $m \in N$ or $r M \subseteq N$. We say that $M$ is a prime module (primary module) if zero submodule of $M$ is prime (primary) submodule of $M$, so $N$ is a prime submodule of $M$ if and only if $M / N$ is a prime module. Moreover, every prime module is primary.

Let $R$ be a ring, and let $N$ be an $R$-submodule of $M$. Then $N$ is pure in $M$ if for any finite system of equations over $N$ which is solvable in $M$, the system is also solvable in $N$. A module is said to be absolutely pure if every embedding of it into any other modules is pure embedding. A submodule $N$ of an $R$-module $M$ is called relatively divisible (or an RD-submodule) if $r N=N \cap r M$ for all $r \in R$. Every RD-submodule of a $P$-secondary module over a commutative ring $R$ is $P$-secondary (see [2, Lemma 2.1]). 
A module $M$ is pure-injective if and only if any system of equations in $M$ which is finitely solvable in $M$, has a global solution in $M$ [7, Theorem 2.8]. The module $N$ is a pure-essential extension of $M$ if $M$ is pure in $N$ and for all nonzero submodules $L$ of $N$, if $M \cap L=0$, then $(M \oplus L) / L$ is not pure in $N / L$. A pure-injective hull $H(M)$ of a module $M$ is a pure essential extension of $M$ which is pure-injective. Every module $M$ has a pure-injective hull which is unique to isomorphism over $M$ [12].

Given an $R$-module $M$ and index set $I$, the direct sum of the family $\left\{M_{i}: i \in I\right\}$ where $M_{i}=M$ for each $i \in I$ will be denoted by $M^{(I)}$. Given a module property $\mathscr{P}$, we will say that a module $M$ is $\sum-\mathscr{P}$ if $M^{(I)}$ satisfies $\mathscr{P}$ for every index set $I$.

Let $R$ be a commutative ring. An element $a \in R$ is said to be regular if there exists $b \in R$ such that $a=a^{2} b$, and $R$ is said to be regular if each of its elements is regular. An important property of regular rings is that every module is absolutely pure (see [13, Theorem 37.6]).

Let $R$ be a ring and $M$ an $R$-module. A prime ideal $P$ of $R$ is called an associated prime ideal of $M$ if $P$ is the annihilator $\operatorname{Ann}(x)$ of some $x \in M$. The set of associated primes of $M$ is written $\operatorname{Ass}(M)$. For undefined terms, we refer to [6, 7].

2. Secondary submodules. In general, a nonzero submodule of a representable (even secondary) $R$-module is not representable (secondary), but we have the following results.

LEMMA 2.1. Let $R$ be a commutative ring and let $0 \neq N$ be an RD-submodule of $R$-module $M$. Then $M$ is $P$-secondary if and only if $N$ and $M / N$ are $P$-secondary.

Proof. If $M$ is $P$-secondary, then $N$ and $M / N$ are $P$-secondary by [2, Lemma 2.1] and [5, Theorem 2.4], respectively. Conversely, suppose that $r \in R$. If $r \in P$, then $r^{n}(M / N)=0$ and $r^{n} N=0$ for some $n$, hence $r^{n} M \subseteq N$ and $0=r^{n} N=r^{n} M \cap N=$ $r^{n} M$. If $r \notin P$, then $r M+N=M, r N=N$, and $N=r N=r M \cap N$, so we have $r M=M$, as required.

COROLLARY 2.2. Let $R$ be a commutative regular ring, and let $0 \neq N$ be a submodule of $R$-module $M$. Then $M$ is $P$-secondary if and only if $N$ and $M / N$ are $P$-secondary.

Proof. This follows from Lemma 2.1.

THEOREM 2.3. Let $R$ be a commutative regular ring. Then every nonzero submodule of a representable $R$-module is representable.

Proof. Let $M$ be a representable $R$-module and let $M=\sum_{i=1}^{n} M_{i}$ be a minimal secondary representation with nilrad $\left(M_{i}\right)=P_{i}$. There is an element $r_{1} \in P_{1}$ such that $r_{1} \notin \cup_{i=2}^{n} P_{i}$. Otherwise $P_{1} \subseteq \cup_{i=2}^{n} P_{i}$, so by [10, Theorem 3.61], $P_{1} \subseteq P_{j}$ for some $j$, and hence $P_{1}=P_{j}$, a contradiction. Thus there exists a positive integer $m_{1}$ such that $r_{1}^{m_{1}} \in \operatorname{Ann}\left(M_{1}\right)$ and the module $r_{1}^{m_{1}} M=\sum_{i=2}^{n} r_{1}^{m_{1}} M_{i}$ is representable. By using this process for the ideals $P_{2}, \ldots, P_{n-1}$, there are integers $m_{2}, \ldots, m_{n-1}$ and elements $r_{2} \in P_{2}, \ldots, r_{n-1} \in P_{n-1}$ such that $s_{n} M=M_{n}$, where $0 \neq s_{n}=r_{1}^{m_{1}} r_{2}^{m_{2}} \cdots r_{n-1}^{m_{n-1}}, s_{n} \in$ $\cap_{i=1}^{n-1} P_{i}$ and $s_{n} \notin P_{n}$. Therefore by a similar argument, there are elements $s_{1}, \ldots, s_{n-1}$ 
such that $M=\sum_{i=1}^{n} s_{i} M$, where for each $i$, where $i=1, \ldots, n, s_{i} \notin P_{i}, s_{i} M=M_{i}$, and $s_{i} \in \cap_{i=1_{i \neq j}}^{n} \operatorname{Ann}\left(M_{j}\right)$.

Let $N$ be a nonzero submodule of $M$ and $0 \neq a \in N$. Then $a=s_{1} b_{1}+\cdots+s_{n} b_{n}$ for some $b_{i} \in M, i=1, \ldots, n$. By assumption, there exists $t_{1}, \ldots, t_{n} \in R$ such that for each $i, s_{i}=s_{i}^{2} t_{i}$. As $0 \neq a, s_{i} b_{i} \neq 0$ for some $i$ and $s_{i} t_{i} a=s_{i}^{2} t_{i} b_{i}=s_{i} b_{i}$, so $s_{i} N \neq 0$. We can assume that $s_{i_{1}} N \neq 0, \ldots, s_{i_{k}} N \neq 0$, where $\left\{i_{1}, \ldots, i_{k}\right\} \subseteq\{1, \ldots, n\}$. By a similar argument as above, if $a \in N$, then $a=\sum_{j=1}^{k} s_{i_{j}} t_{i_{j}} a \in \sum_{j=1}^{k} s_{i_{j}} N$, and hence $N=\sum_{j=1}^{k} s_{i_{j}} N$. Since for each $j$, where $j=1, \ldots, k, s_{i_{j}} N$ is pure in the $P_{i_{j}}$-secondary module $M_{i_{j}}$, it is $P_{i_{j}}$-secondary by [2, Lemma 2.1], as required.

THEOREM 2.4. Let $R$ be a commutative ring and let $N$ be a prime submodule of secondary $R$-module of $M$. Then $N$ is $(N: M)$-secondary.

Proof. Suppose that $M$ is a $P$-secondary module over $R$. Let $r \in R$. If $r \in P$, then $r^{n} N \subseteq r^{n} M=0$ for some $n$. If $r \notin P$, then $r M=M$. Suppose that $n \in N$, so there is an element $m \in M$ such that $n=r m$. As $N$ is a prime submodule of $M$ and $N \neq r M=M$, $m \in N$, so $r N=N$, hence $N$ is $P$-secondary.

By [4, Lemma 1], the ideal $P^{\prime}=(N: M)=\{r \in R: r M \subseteq N\}$ is prime. Clearly, $P^{\prime} \subseteq P$. Let $s \in P$. Then $s^{n} N=s^{n} M=0$ for some $n$. There is an element $m \in M$ such that $m \notin N$ and $s^{n} m=0 \in N$, so $s^{n} \in P^{\prime}$, hence $s \in P^{\prime}$. Thus $P=P^{\prime}$, as required.

Proposition 2.5. Let $R$ be a commutative ring and let $N$ be a prime submodule of $P$-second $R$-module of $M$. Then $N$ is an RD-submodule of $M$.

Proof. Let $r \in R$. If $r \in P$, then $r N \subseteq r M=0$, so $r N=N \cap r M=0$. If $r \notin P$, then $r M=M$, so the homothety $M / N \stackrel{r}{\rightarrow} M / N$ is not zero since $N$ is prime. It follows that the above homothety is injective. If $a \in N \cap r M$, then there is $b \in M$ such that $a=r b$. Since $r(b+N)=0$, so $b \in N$, hence $r N=N \cap r M$, as required.

THEOREM 2.6. Let $M$ be a $P$-second module over a commutative ring $R$, and let $N$ be a prime submodule of $M$. Then every submodule of $M$ properly containing $N$ is an $\mathrm{RD}$-submodule. In particular, it is P-second.

Proof. Let $K$ be a submodule of $M$ properly containing $N$. Then $K / N$ is a prime submodule of prime and $P$-second module $M / N$, so by Proposition $2.5, K / N$ is an RDsubmodule of $M / N$. Now the assertion follows from [3, Consequences 18-2.2(c)] and Proposition 2.5.

LEMMA 2.7. Let $M$ be a nonzero module over a commutative domain $R$. Then $M$ is (0) -second if and only if $M$ is (0)-secondary.

Proof. The proof is completely straightforward.

By [3, Proposition 11-3.11] and [11, Proposition 12, page 506] (see also [14]), and the definitions of secondary and primary modules, we obtain the following corollary.

COROLlaRY 2.8. Let $R$ be a commutative ring.

(i) Every Artinian primary module over $R$ is secondary.

(ii) Every Noetherian secondary module over $R$ is primary.

(iii) Every finitely generated secondary module is primary. 
LEMMA 2.9. Let $R$ be a commutative ring. Let $K$ and $N$ be submodules of an $R$-module $M$ such that $N$ is prime and $K$ is $P$-secondary. Then $N \cap K$ is $P$-secondary.

Proof. Let $r \in R$. If $r \in P$, then $r^{n}(N \cap K) \subseteq r^{n} K=0$ for some $n$. Suppose $r \notin P$ and $t \in N \cap K$. Then $t=r s$ for some $s \in K$ since $K P$-secondary. As $N$ is prime, we have $s \in N$, and hence $t \in r(N \cap K)$. This gives, $N \cap K=r(N \cap K)$.

THEOREM 2.10. Let $M$ be a representable module over a commutative ring $R$, and let $N$ be a prime submodule of $M$ with $(N: M)=P$. Then the following hold:

(i) $N$ is representable;

(ii) $M / N$ is P-secondary.

Proof. (i) Let $M$ be a representable $R$-module and let $M=\sum_{i=1}^{m} M_{i}$ be a minimal secondary representation with nilrad $\left(M_{i}\right)=P_{i}$. For each $i, i=1,2, \ldots, m$, let $m_{i} \in M_{i}$ and $r_{i} \in P_{i}$. Then $r_{i}^{n_{i}} m_{i}=0$ for some $n_{i}$, and we have $\left(r_{i}^{n_{i}}+P\right)\left(m_{i}+M_{i}\right)=0$ and hence either $P_{i} \subseteq P$ or $M_{i} \subseteq N(i=1,2, \ldots, m)$. It follows that $M_{i} \nsubseteq N$ for some $i$ (otherwise $M=N$ ). If $M_{i} \nsubseteq N$ and $M_{j} \nsubseteq N$ for $i \neq j$, then $P=P_{i}=P_{j}$, a contradiction (for if $t \in P-P_{i}$ then $M_{i}=t M_{i} \subseteq t M \subseteq N$ ). Therefore, without loss of generality, we can assume that $M_{1} \nsubseteq N$ and $M_{i} \subseteq N$, so $P_{1}=P$ and $P_{i} \nsubseteq P(i=2,3, \ldots, m)$. Then $M_{2}+M_{3}+\cdots+M_{m} \subseteq N$ and

$$
N=N \cap M=N \cap\left(M_{1}+\cdots+M_{m}\right)=M_{2}+\cdots+M_{m}+\left(N \cap M_{1}\right) .
$$

Now the assertion follows from Lemma 2.9.

(ii) Since $M=M_{1}+N$, we have $M / N=\left(M_{1}+N\right) / N \cong M_{1} /\left(M_{1} \cap N\right)$, as required.

Proposition 2.11. Let $R$ be a Dedekind domain, and let $M$ be a $0 \neq P$-secondary $R$-module. Then $M$ is a P-primary module.

Proof. Let $r \in R$. If $r \in P$, then the homothety $M \stackrel{r \cdot}{\longrightarrow} M$ is nilpotent since $M$ is secondary. Suppose that $r \notin P$. If $r a=0$ for some $0 \neq a \in M$, then by [6, Theorem 6.1], there exists $0 \neq b \in M$ and $Q \in \operatorname{Ass}(M)$ such that $r \in Q$ and $Q=\left(0:_{R} b\right)$. As $(0: M) \subseteq(0: b)=Q$, we have $P=Q$, a contradiction. So the homothety $M \stackrel{r}{\longrightarrow} M$ is injective, as required.

REMARKS. (i) Let $R$ be a domain which is not a field. Then $R$ is a prime $R$-module (since $R$ is torsion-free) but it is not secondary (even it is not pure-injective).

(ii) Let $R$ be a local Dedekind domain with maximal ideal $P=R p$. We show that the module $E(R / P)$ is not prime (but it is (0)-secondary). Set $E=E(R / P)$ and $A_{n}=\left(0:_{E}\right.$ $\left.P^{n}\right)(n \geq 1)$. Then by [2, Lemma 2.6], $P A_{n+1}=A_{n}, A_{n} \subseteq E$ is a cyclic $R$-module with $A_{n}=R a_{n}$ such that $p a_{n+1}=a_{n}$, every nonzero proper submodule $L$ of $E$ is of the form $L=A_{m}$ for some $m$ and $E$ is Artinian module with a strictly increasing sequence of submodules

$$
A_{1} \subset A_{2} \subset \cdots \subset A_{n} \subset A_{n+1} \subset \cdots
$$

We claim that $\left(A_{n}:_{R} E\right)=0$ for every $n$. Suppose that $r \in\left(A_{n}:_{R} E\right)$ with $r \neq 0$. Then $r E \subseteq A_{n}$ and for all $a \in M$, we have $a=r b$ for some $b \in M$ since $E$ is injective (= divisible). Thus $a=r b \in A_{n}$, so $E=A_{n}$, a contradiction. Therefore $\left(A_{n}:_{R} E\right)=0$ for 
every integer $n \geq 1$. However no $A_{n}$ is a prime submodule of $E$, for if $m$ is any positive integer, then $p^{m} \notin\left(A_{n}:_{R} E\right)=0$ and $a_{n+m} \notin A_{n}$, but $p^{m} a_{m+n}=a_{n} \in A_{n}$.

THEOREM 2.12. Let $R$ be a Dedekind domain, and let $M$ be an $R$-module. Then $M$ is $0 \neq P$-second if and only if $M$ is $P$-prime.

Proof. By Proposition 2.11, it is enough to show that if $M$ is $P$-prime, then $M$ is $P$-second. Since $(0: M)=P$ is a maximal ideal in $R$, so $M$ is a vector space over $R / P$, hence $M$ is $P$-second.

Proposition 2.13. Let $R$ be a Dedekind domain. Then any $0 \neq P$-prime $R$-module is a direct sum of copies of $R_{P} / P R_{P} \cong R / P$.

Proof. By the proof of Proposition 2.11, every element of $R-P$ acts invertibly on $M$, so the $R$-module structure of $M$ extends naturally to a structure of $M$ as a module over the localisation $R_{P}$ of $R$ at $P$. Therefore, we can assume that $R$ is a commutative local Dedekind domain with maximal ideal $P=R p$. Let $M_{j}$ denote the indecomposable summand of $M$, so $M_{j}$ is $P$-prime. Let $m_{j}$ be a nonzero element of $M_{j}$, hence $\left(0: m_{j}\right)=$ $(0: M)=P$. Then $R m_{j} \cong R / P$ is pure in $M_{j}$ since $m_{j}$ is not divisible by $p$ in $M_{j}$, but by [1, Proposition 1.3], the module $R / P$ is itself pure-injective, so $R m_{j}$ is a direct summand of $M_{j}$, and hence $M_{j} \cong R m_{j}$, as required.

\section{Pure-injective modules}

Proposition 3.1. Let $M$ be a $P$-secondary module over a commutative ring $R$. Then $H=H(M)$, the pure-injective hull, is $P$-secondary.

PRoof. Let $r \in R$. If $r \notin P$, then $r M=M$, so $M$ satisfies the sentence for all $x$ there exists $y(x=r y)$, and hence so does $H$ (because any module and its pure-injective hull satisfy the same sentences [7, Chapter 4]). If $r \in R$, then $r^{n} M=0$, so $M$ satisfies the sentence for all $x\left(r^{n} x=0\right)$, hence so does in $H$, as required.

THEOREM 3.2. The following conditions are equivalent for a Prufer domain R:

(i) the ring $R$ is a Dedekind domain;

(ii) every secondary $R$-module is pure-injective.

Proof. Let $R$ be a Dedekind domain and $M$ a secondary $R$-module. If $\operatorname{Ann}(M)=0$, then $M$ is divisible, hence injective. If $\operatorname{Ann}(M) \neq 0$, then $M$ is a torsion $R$-module of bounded order, so that $M$ is $\Sigma$-pure-injective (see [15]). In both cases, $M$ is $\Sigma$-pureinjective (so pure-injective).

Conversely, let $R$ be a Prufer domain with the property that every secondary module is pure-injective. In order to prove that $R$ is Dedekind domain, it suffices to show that every divisible $R$-module is injective. Let $M$ be a divisible $R$-module. Then $M$ is secondary, Hence pure-injective. Since $R$ is Prufer, pure-injective modules are RDinjective (see [7]). The embedding of $M$ in its injective envelope $E(M)$ is an RD-pure monomorphism, because for every nonzero $r \in R$ we have that $M=r M$, so that $r E(M) \cap M \subseteq M \subseteq r M$. Since $M$ is the RD-injective, $M$ is a direct summand of $E(M)$. Thus $M$ is injective. This shows that $R$ is a Dedekind domain. 
REMARKS. (i) There is a module over a commutative regular ring which is injective but not secondary (see [9, Theorem 2.3]). The commutative regular ring $R=F \times F$, $F$ a field, is an Artinian Gorenstein, that is, $R$ is injective (so pure-injective) as an $R$ module. But $R$ is not secondary, because multiplication by $(1,0)$ is neither nilpotent nor surjective.

(ii) The above consideration thus leads us to the following question: are secondary modules pure-injective? The answer is yes because of the following reason. Every non-Noetherian Prufer domain has secondary modules that are not pure-injective. For instance, every non-Noetherian valuation domain has secondary modules that are not pure-injective.

Proposition 3.3. Let $M$ be an $R$-module.

(i) $M$ is $\sum$-secondary if and only if $M$ is secondary.

(ii) Let $M$ be a direct sum of modules $M_{i}(i \in I)$ where for each $i, M_{i}$ is secondary and $\operatorname{Ann}\left(M_{i}\right)=\operatorname{Ann}\left(M_{j}\right)$ for all $i, j \in I$. Then $M$ is secondary.

Proof. (i) The necessity is immediate by the definition. Conversely, suppose that $M$ is $P$-secondary. Given an index set $J$, and let $r \in R$. If $r \in P$, then $r^{n} M=0$ for some $n$, so $r^{n} M^{(J)}=0$. If $r \notin P$ then $r M=M$, so $r M^{(J)}=M^{(J)}$, as required.

(ii) Since the annihilators of all direct summands coincide, we can assume that $M_{i}$ is $P$-secondary (say) for all $i \in I$. Now the proof of (ii) is similar to that (i) and we omit it.

COROLLARY 3.4. Let $M$ be an indecomposable $\Sigma$-pure-injective module over a commutative Prufer ring $R$. Then $M$ is secondary.

Proof. Set $P=\left\{r \in R: \operatorname{Ann}_{M} r \neq 0\right\}$ and $P^{\prime}=\cap_{n} P^{n}$. Then $P$ and $P^{\prime}$ are prime ideals in $R$ by [8, Fact 3.1 and Lemma 2.1]. By [8, Fact 3.2], $M$ is either $P$-secondary or $P^{\prime}$-secondary, as required.

COROLLARY 3.5. Every $\Sigma$-pure-injective module over a Prufer ring is representable.

Proof. Suppose $M$ is a $\Sigma$-pure-injective module over a commutative Prufer ring $R$. By [8, page 967], we can write $M=M_{1} \oplus \cdots \oplus M_{m}$ where $M_{i}$ is secondary for all $i$ by Proposition 3.3 and Corollary 3.4, as required.

ACKNOWLEDGMENT. The author thanks the referee for useful comments.

\section{REFERENCES}

[1] S. E. Atani, On pure-injective modules over pullback rings, Comm. Algebra 28 (2000), no. 9, 4037-4069.

[2] _ On secondary modules over Dedekind domains, Southeast Asian Bull. Math. 25 (2001), no. 1, 1-6.

[3] J. Dauns, Modules and Rings, Cambridge University Press, Cambridge, 1994.

[4] S. M. George, R. L. McCasland, and P. F. Smith, A principal ideal theorem analogue for modules over commutative rings, Comm. Algebra 22 (1994), no. 6, 2083-2099.

[5] I. G. Macdonald, Secondary representation of modules over a commutative ring, Symposia Mathematica, Vol. 11 (Convegno di Algebra Commutativa, INDAM, Rome, 1971), Academic Press, London, 1973, pp. 23-43. 
[6] H. Matsumura, Commutative Ring Theory, Cambridge Studies in Advanced Mathematics, vol. 8, Cambridge University Press, Cambridge, 1986.

[7] M. Prest, Model Theory and Modules, London Mathematical Society Lecture Note Series, vol. 130, Cambridge University Press, Cambridge, 1988.

[8] M. Prest and G. Puninski, $\Sigma$-pure-injective modules over a commutative Prüfer ring, Comm. Algebra 27 (1999), no. 2, 961-971.

[9] R. Y. Sharp, Secondary representations for injective modules over commutative Noetherian rings, Proc. Edinburgh Math. Soc. (2) 20 (1976), no. 2, 143-151.

[10] - Steps in Commutative Algebra, London Mathematical Society Student Texts, vol. 19, Cambridge University Press, Cambridge, 1990.

[11] W. V. Vasconcelos, On finitely generated flat modules, Trans. Amer. Math. Soc. 138 (1969), 505-512.

[12] R. B. Warfield Jr., Purity and algebraic compactness for modules, Pacific J. Math. 28 (1969), 699-719.

[13] R. Wisbauer, Foundations of Module and Ring Theory, Algebra, Logic and Applications, vol. 3, Gordon and Breach Science Publishers, Pennsylvania, 1991.

[14] S. Yassemi, The dual notion of prime submodules, to appear in Arch. Math.

[15] W. Zimmermann, Rein injektive direkte Summen von Moduln, Comm. Algebra 5 (1977), no. 10, 1083-1117 (German).

Shahabaddin Ebrahimi Atani: Department of MATHEMATICS, University of GUILAN, P.O. BOX 1914, RASHT, IRAN

E-mail address: ebrahimi@cd.gu.ac.ir 


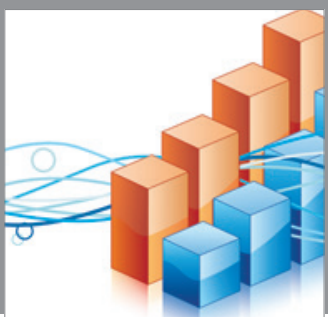

Advances in

Operations Research

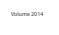

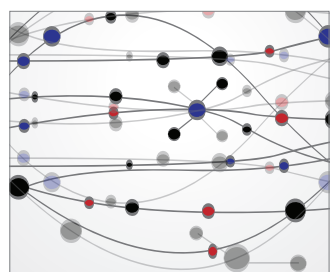

\section{The Scientific} World Journal
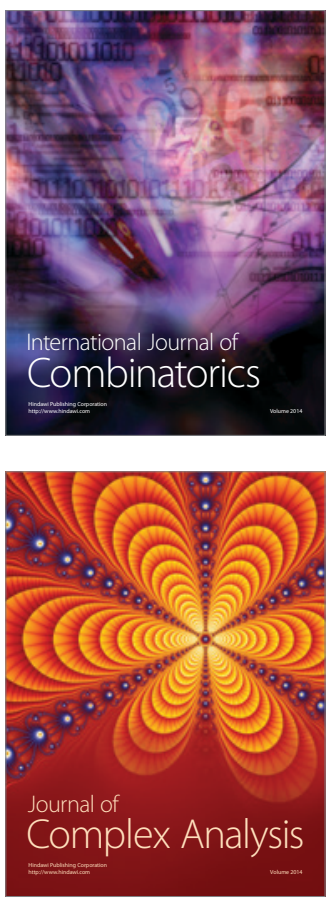

International Journal of

Mathematics and

Mathematical

Sciences
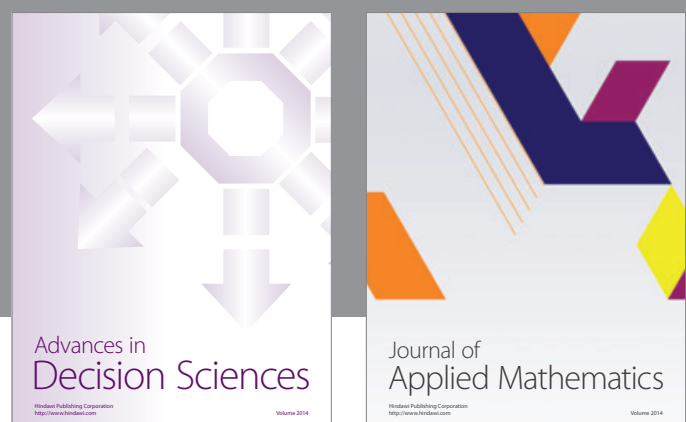

Journal of

Applied Mathematics
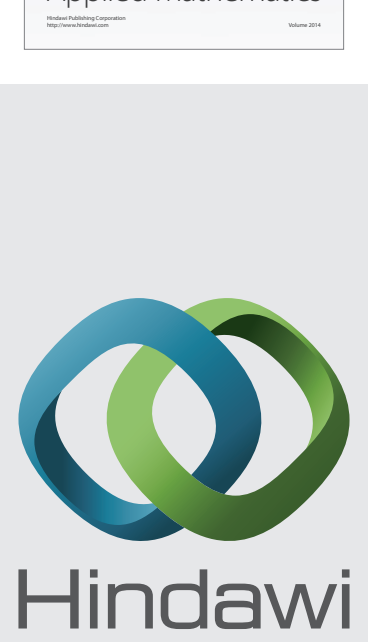

Submit your manuscripts at http://www.hindawi.com
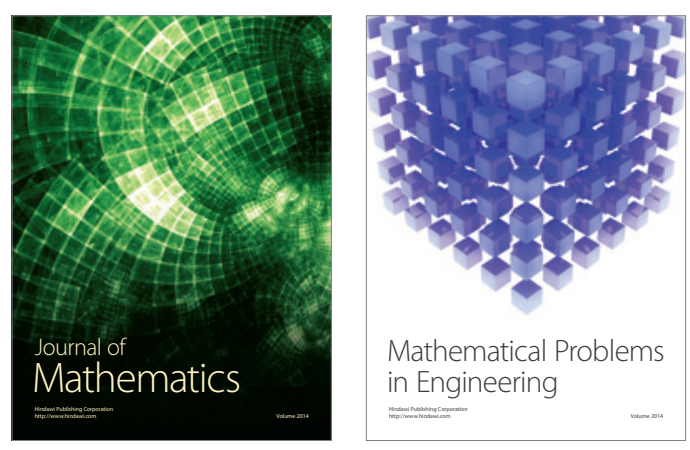

Mathematical Problems in Engineering
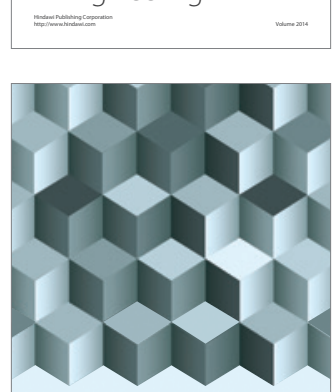

Journal of

Function Spaces
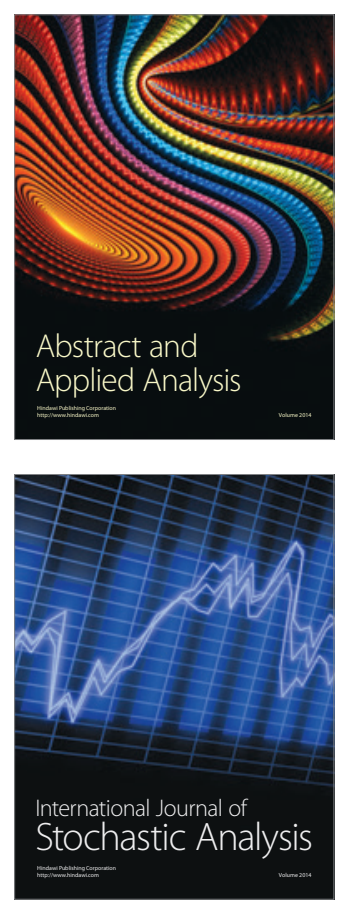

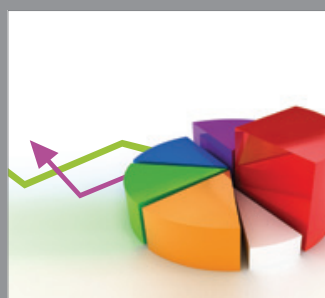

ournal of

Probability and Statistics

Promensencen
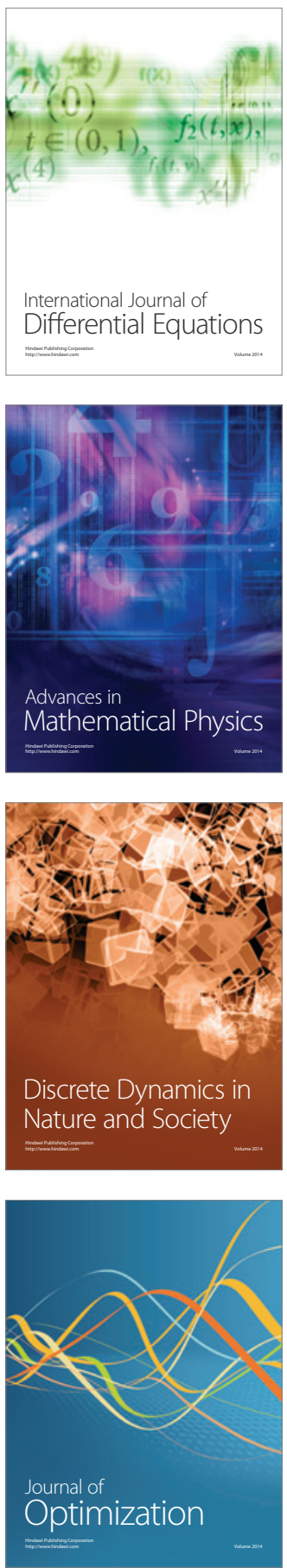